\title{
Redes Multidifusión utilizando Conmutación de Etiquetas Multiprotocolo y Estándares de Señalización
}

\author{
Danilo A. López, Cesar A. Hernández* y Octavio J. Salcedo \\ Universidad Distrital Francisco José de Caldas, Ingeniería Electrónica, Grupo de investigación \\ Internet Inteligente, Cra 7 No 40 - 53, piso 7, Bogotá D.C.-Colombia. \\ (e-mail: \{dalopezs, cahernandezs, ojsalcedop\}@udistrital.edu.co).
}

* Autor a quien debe ser dirigida la correspondencia.

Recibido Mar. 16, 2012; Aceptado May. 24, 2012; Versión final recibida Jul. 11, 2012

\section{Resumen}

Este artículo expone los resultados de una evaluación del sistema de conmutación de etiquetas multiprotocolo (MPLS) como tecnología multidifusión (envío de datos desde una fuente a varios usuarios) utilizando el protocolo de reserva de recursos con extensiones de ingeniería de tráfico (RSVP-TE). Para determinar las ventajas de ésta implementación, el sistema se compara con el funcionamiento de una red de transmisión de data-gramas IP basada en el estándar independiente multienvío en modo denso (PIM-DM). Las simulaciones son realizadas utilizando los paquetes de software OPNET y GNS-3. Se concluye que la conmutación de etiquetas MPLS utilizando RSVP-TE posee ventajas muy superiores a otras arquitecturas. Dichas ventajas incluyen una respuesta más rápida ante fallas en los enlaces, menor retardo y fluctuación y la posibilidad de reservar ancho de banda para tráfico con prioridad.

Palabras clave: redes multidifusión, conmutación de etiquetas, estándares de señalización, ingeniería de tráfico

\section{Multicast Network Performance using Multiprotocol Label Switching Together with Signaling Standards}

\begin{abstract}
The present article shows the results of the evaluation of Multiprotocol Label Switching (MPLS) as a technology for the transfer of multicast traffic (sending data from one source to several users) through the use of signaling protocols such as RSVP-TE. To identify the advantages of such an implementation, the setup in this work is compared to a multicast IP network based on the PIMDM protocol. Simulations were carried out using software packages such as OPNET and GNS-3. It was found that MPLS+RSVP-TE offers greater advantages over other architectures. Some of these advantages include quicker link-failure response, shorter delays and less jitter, and also the possibility to reserve bandwidth for priority traffic.
\end{abstract}

Keywords: multicast network, multiprotocol label switching, signaling standards, traffic engineering 


\section{INTRODUCCIÓN}

La tecnología Multicast o de multidifusión, nace como respuesta a la necesidad de enviar grandes volúmenes de información realizando una única transmisión a diferentes usuarios simultáneamente, lo que permite una disminución en el tráfico de la red, optimizando el ancho de banda utilizado. Para realizar este tipo de transferencias se utiliza el protocolo de mensajes de grupos de Internet (Islam y Atwood, 2006) en donde cada host informa su pertenencia al dominio enviando mensajes de unión al encaminador multienvio. No obstante, el protocolo de Internet (IP) no tiene la posibilidad de seleccionar diferentes niveles de calidad de servicio (Thomas y Ali, 2010) para los distintos tipos de aplicaciones de usuario, lo que significa que compiten por el mismo ancho de banda un correo electrónico, un archivo que se descarga desde un servidor de transferencia de archivos y una videoconferencia.

Las características del protocolo IP mencionadas anteriormente no permiten el envío de datos multidifusión con requerimientos de calidad de acuerdo a la aplicación. Por esta razón se hace necesaria la adición de estructuras de siguiente generación como MPLS (multiprotocolo de conmutación de etiquetas) que permitan entre otros, priorizar el tráfico para el transporte de flujos de datos en tiempo real, reservar ancho de banda garantizando un transporte fiable. El principal aporte de este trabajo se centra en la evaluación del comportamiento de la integración del protocolo de reservación de recursos con ingeniería de tráfico (RSVP-TE) asociado con MPLS en la transmisión de trazas reales de video y voz en tiempo real, frente al ofrecido por PIM-DM en el núcleo de una red de área extensa. En cada escenario se busca determinar cómo se desempeña cada tecnología a nivel de rendimiento analizando métricas tales como variabilidad, latencia, tiempos de respuesta y pérdidas de paquetes, haciendo uso de herramientas computacionales.

\section{ESTADO DEL ARTE}

Se ha podido establecer que la investigación desarrollada en MPLS aplicada a redes con tráfico multicast ha ido evolucionando, pero aún quedan muchas incógnitas por resolver entre las que se encuentra su comportamiento para aplicar balanceo de carga en este tipo de entornos, el desempeño para la transmisión de aplicaciones multidifusión con tecnologías de señalización (Yuanzhe, 2011). Puntualmente en este sentido (Metz, 2006), hace una propuesta denominada ERM (encaminador de borde multienvío) donde se busca reducir el establecimiento del intercambio de rutas por etiquetas y la asignación de flujos multidifusión a un problema de tipo unidifusión. Para lograr esto, el objetivo es que únicamente los direccionadores de borde (nodos) del dominio MPLS sean los puntos de ramificación del árbol de distribución multicast. El Autor realiza varias simulaciones y sustenta el buen funcionamiento de ERM analizando la saturación de los enlaces y los retardos presentados.

De otro lado, (Hachimi y Abouaissa, 2006) determinan la necesidad de la creación de un nuevo protocolo para la transferencia de tráfico multidifusión sobre MPLS, para lo cual proponen el protocolo de árbol multicast (MMT), que utiliza caminos de conmutación de etiquetas (LSP) entre los encaminadores que presentan ramificaciones del árbol para reducir sus estados e incrementar escalabilidad. Para comprobar y evaluar la eficiencia del protocolo, presentan simulaciones sobre el simulador de redes NS, donde concluyen que puede obtenerse un tiempo de procesamiento de paquetes más rápido dadas las características de intercambio de etiquetas de MPLS.

\section{SIMULACIONES}

\section{Escenarios de simulación}

Se utilizaron dos escenarios de simulación, el primero con PIM-DM y el segundo con MPLS RSVP-TE. La topología en ambos casos es la misma y es mostrada en la Fig. 1, donde los enlaces seriales tienen un ancho de banda de $1544 \mathrm{Kbps}$ (T1). Igualmente se muestra el transmisor, el servidor de sincronización de la red (NTP) y los receptores, además se especifica la función de cada encaminador dentro de la nube MPLS. Las simulaciones realizadas incluyen 
pruebas de respuesta ante la caída de enlaces, reserva de recursos y medición de la fluctuación y retardo, tanto para la red PIM-DM como para la nube MPLS.

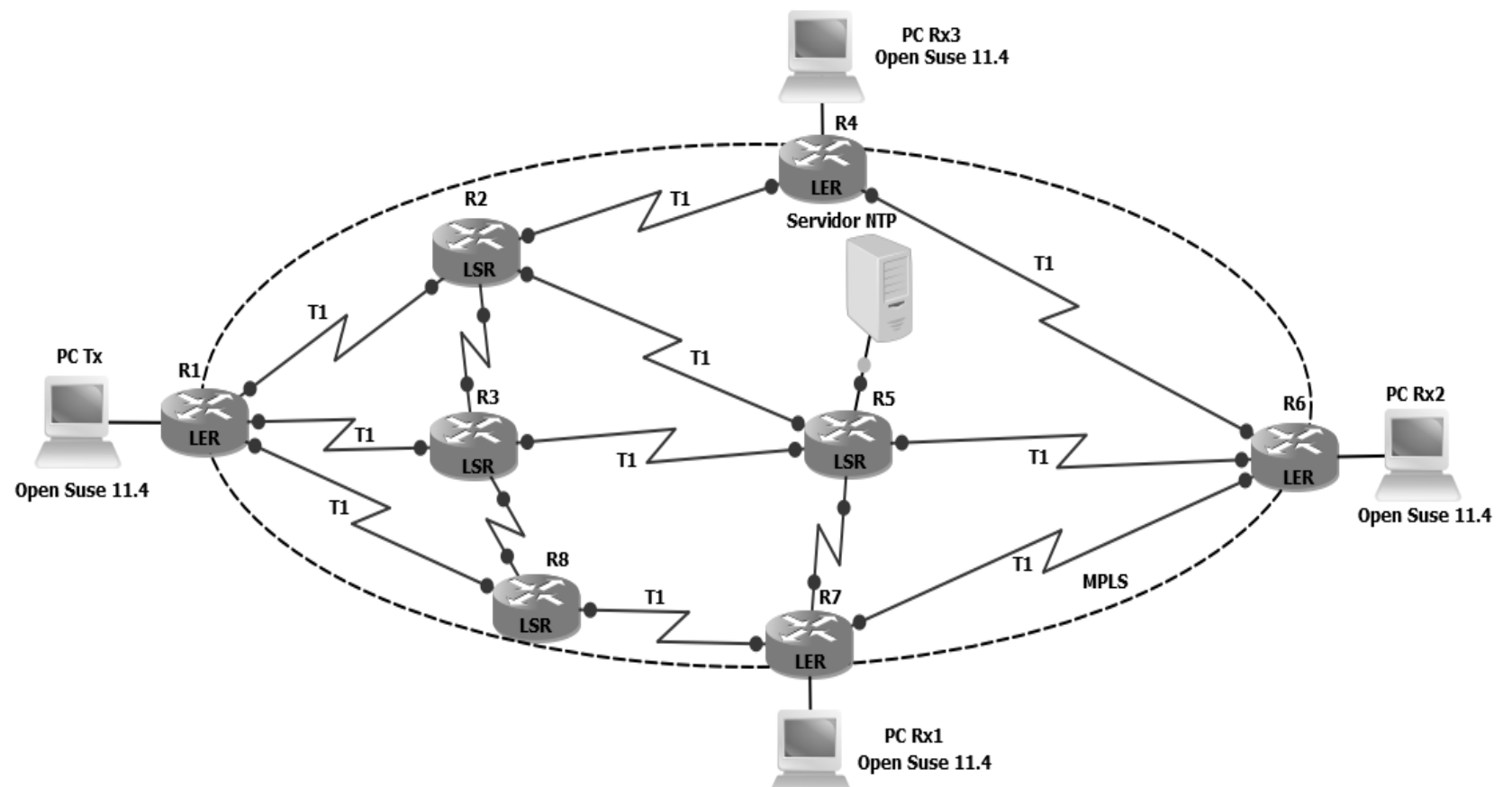

Fig. 1: Topología de la red simulada.

\section{RESULTADOS}

Comportamiento ante la caída de enlaces

Para observar el comportamiento de los dos escenarios ante la caída de un enlace, se generó tráfico de video conferencia a $30 \mathrm{~KB} / \mathrm{seg}$ hacia el grupo multidifusión desactivando el enlace entre el nodo R1 y R8, que hace parte de la ruta entre el transmisor y el primer equipo receptor Rx1. La Fig. 2 muestra el tráfico que recibe el dicho receptor, obtenido mediante el analizador de redes Wireshark, (2011).
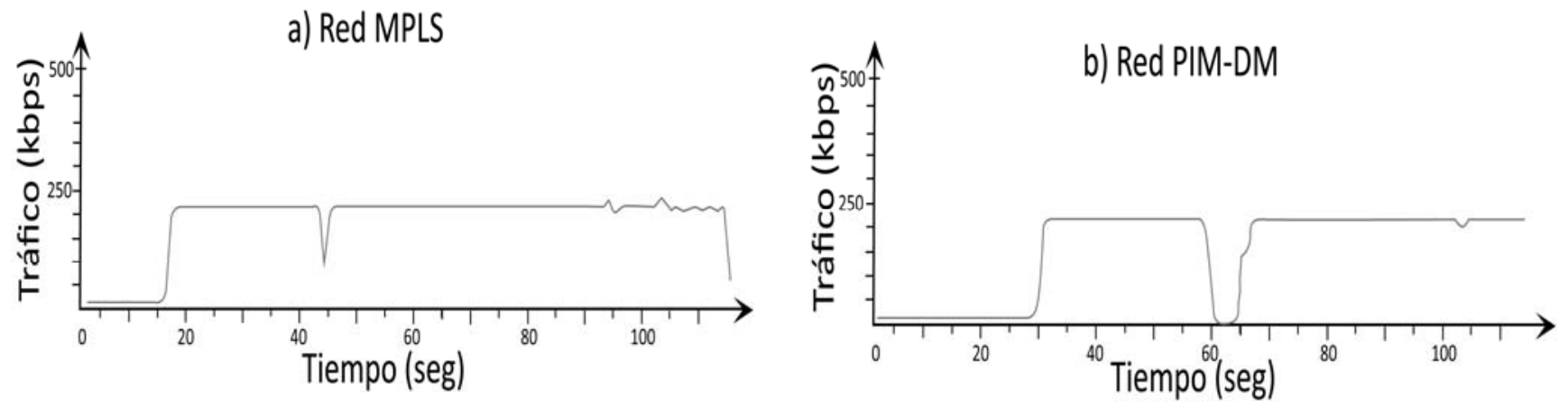

Fig. 2: Comparación entre el tiempo de respuesta ante la caída de un enlace. a) Red MPLS. b) Red PIM-DM.

Tal y como se observa, en la red PIM-DM, hay un tiempo específico (alrededor de 7 segundos) antes de que vuelva a establecerse una nueva ruta para continuar con el envío. Esto se debe al tiempo que tarda el protocolo de enrutamiento en detectar el cambio en la topología, recalcular el algoritmo de Dijkstra, y establecer una base de datos en el encaminador para que PIM-DM pueda construir el nuevo árbol de distribución hacia los destinos. 
Para el caso de la red MPLS con ingeniería de tráfico, el tiempo de recuperación es mucho más corto, gracias a la creación de túneles de respaldo para los enlaces principales; tan pronto como se detecta una cambio en la topología, fast-reroute (Hailong et al., 2009) permite una rápida conmutación a dichos túneles, logrando que no se pierda tráfico mientras se recalculan nuevos caminos hacia los destinatarios. Esta detección se realiza a través de los mensajes Hello de RSVP, los cuales advierten inmediatamente a los nodos adyacentes acerca de algún fallo, facilitando el trabajo de re-encaminamiento (Thomas y Ali, 2010) para poder continuar con la prestación del servicio.

Para evaluar de manera más detallada el resultado de la prueba realizada, se graficaron las pérdidas totales en el receptor con ayuda de MGEN (Networks and communication systems branch, 2008) (Fig. 3). Claramente, para la red IP se tiene un porcentaje mayor de pérdidas con respecto a la red de conmutación de etiquetas. Esto permite concluir acerca del buen comportamiento que presenta MPLS para recuperarse ante fallas.

a) Red MPLS

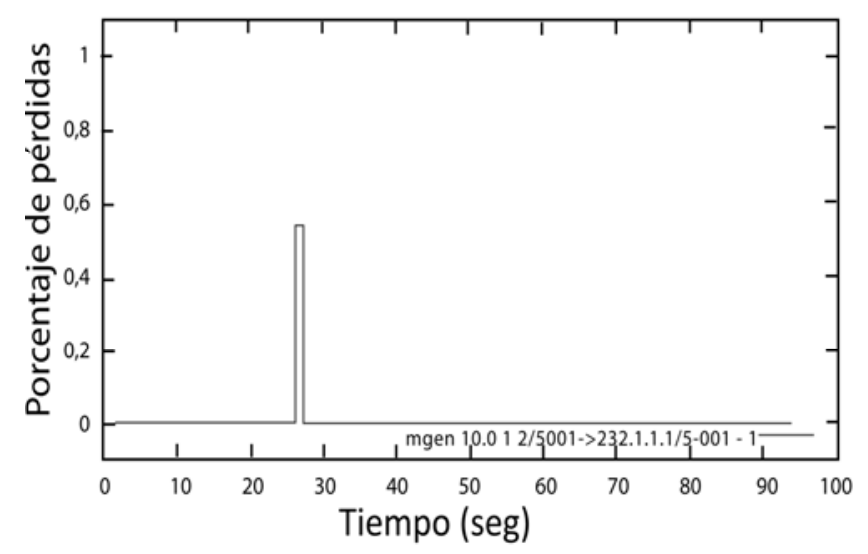

b) Red PIM-DM

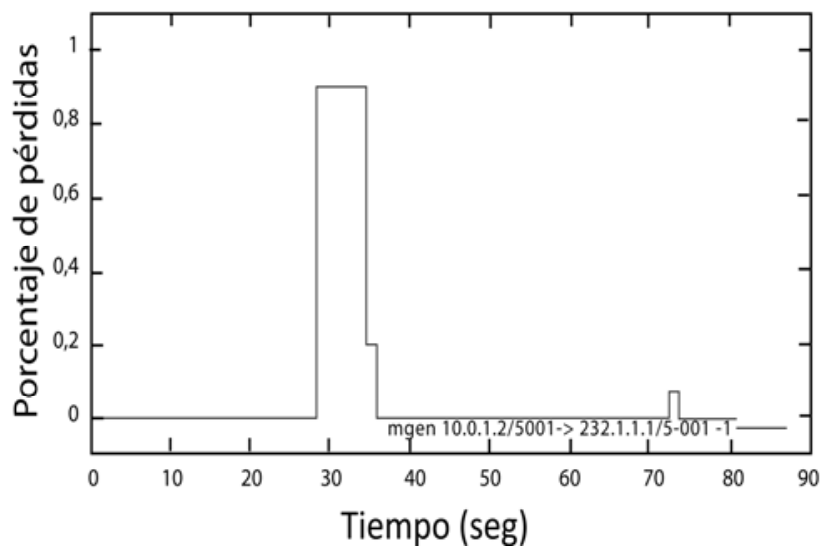

Fig. 3: Porcentaje de pérdidas en el receptor luego de haber generado la falla en el enlace R1-R8. a) Red MPLS b) Red IP.

\section{Reserva de Recursos}

Otra importante ventaja que añaden las redes basadas en el estándar MPLS, es la garantía de un ancho de banda disponible a lo largo de una trayectoria. Para esto se hace necesario la inclusión de una estructura de señalización como RSVP-TE que se encargue de realizar la reserva de recursos y la distribución de etiquetas para cada camino de conmutación. Además, se debe incluir un algoritmo que sea capaz de encontrar las rutas que cumplan con las restricciones establecidas por el administrador, como el estándar de la ruta más corta. Esta característica permite que cada camino virtual configurado para cada par fuente-grupo $(S, G)$, disponga de un rango de frecuencias exclusivo para él.

Para analizar el rendimiento dado por estas características, se configuraron dos grupos multienvio en cada escenario, manteniendo los mismos receptores y al nodo Tx como fuente. De esta manera, deben existir dos árboles de distribución para cada grupo multidifusión asociados al origen de los datos. El envió tráfico multicast hacia los dos grupos, se realizó emitiendo paquetes de video a 1024 bytes a una tasa de 100 paquetes por segundo. Los resultados se detallan en la Fig. 4, donde se observa que la red MPLS no ha presentado ningún paquete perdido, mientras que en la red PIM-DM existe un porcentaje considerable de pérdidas. Gracias a la reserva de recursos aplicada mediante RSVP-TE, cada LSP tiene el ancho de banda suficiente para transmitir el flujo de datos proveniente de la fuente. Así, el tráfico fluye sin inconvenientes, evitando el descarte de datos debido a posibles encolamientos en los nodos.

Como varios LSP pueden elegir rutas que no son las más cortas hacia los destinos, el flujo sensible a retardos (como por ejemplo la transmisión de video) puede verse afectado. Este 
problema se resuelve al establecer una prioridad más alta a uno de los túneles, de manera que este tipo de datos tome inicialmente la ruta más corta, y luego se calculen los caminos para los demás LSPs. También se pueden definirse de manera estática los caminos de conmutación de etiquetas que transportan datos de alta prioridad, permitiendo que el administrador elija por donde debe viajar la información, facilitando el control, gestión y solución de problemas de red.

a) Red MPLS

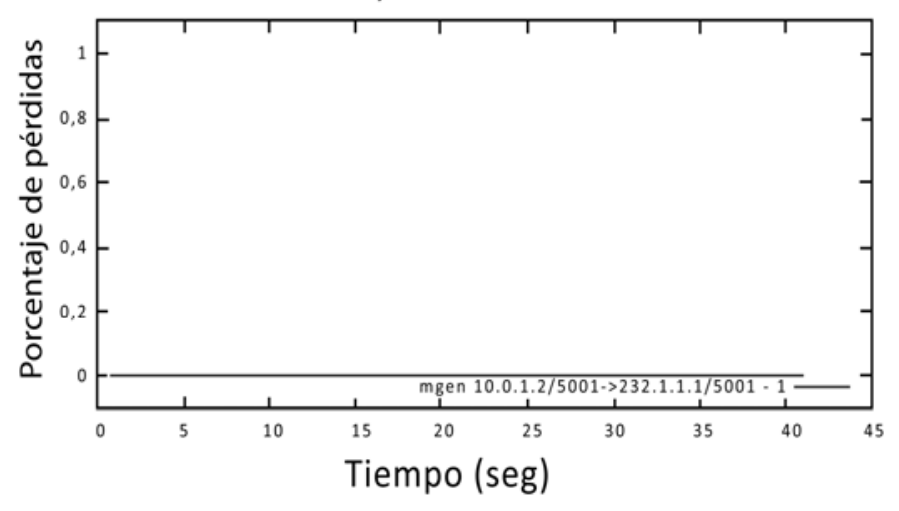

b) Red PIM-DM

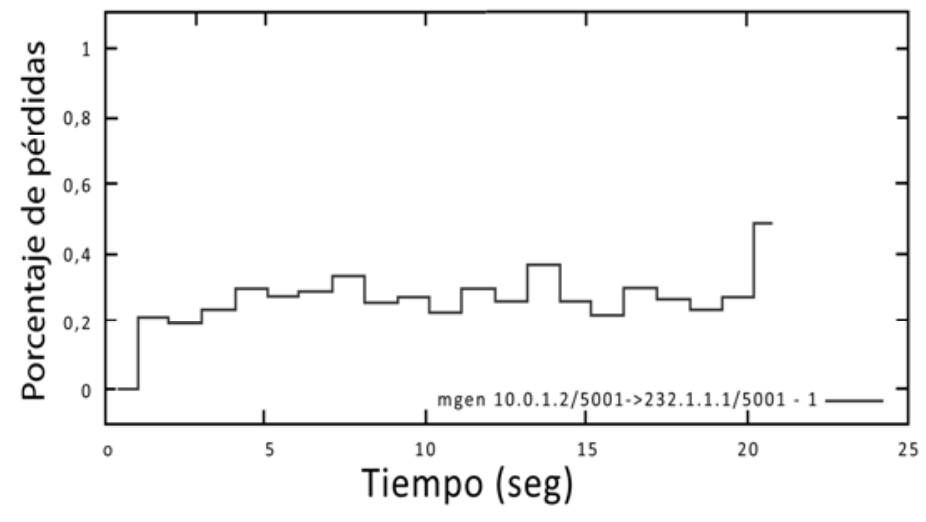

Fig. 4: Porcentaje de pérdidas para un receptor del grupo multicast 232.1.1.1. a) Red MPLS b) Red PIM-DM.

\section{Latencia}

Para este caso, se utilizaron trazas idénticas de video (a 160Kbits/seg durante 30 segundos) en ambos escenarios para el grupo multidifusión 232.1.1.1, las cuales se recibieron en los receptores $\mathrm{R} \times 1$ y $\mathrm{R} \times 2$, obteniendo lo presentado en las Fig. 5 y 6 , para cada caso. Para obtener resultados precisos, se sincronizaron las máquinas haciendo uso de un servidor NTP.

Tras realizar varias mediciones se obtuvieron promedios de la latencia en ambas redes; para el receptor Rx2, generalmente IP arrojaba valores alrededor de los 37,1 mseg, comparado con los 32,7 mseg que caracterizaban el segundo escenario. Para el caso de Rx1, IP tenía promedios de 41,4 mseg contra los 35,8 mseg de MPLS. Las diferencias no resultan significativas dado que la topología es pequeña y el número de saltos entre el transmisor y los receptores es apenas 2 . Sin embargo, en redes mucho más extensas las diferencias de conmutación a nivel de capa de enlace de datos harán que PIM-DM obtenga niveles muchos más altos que los observados en un entorno de conmutación de etiquetas.

\section{Fluctuación}

Se utilizó la aplicación Mausezahn (Lodi et al., 2006), para obtener un análisis de la fluctuación emulando una transmisión de voz (Amir et al., 2006) a una tasa de 64 kilobits por segundo a través del protocolo de transporte en tiempo real (RTP), utilizando codificación G.711 (Sung y Lin, 
2008). La Fig. 7, describe la fluctuación encontrada para el receptor $R \times 1$, de la que se puede concluir que para los 2 casos, hay un alto número de paquetes con un valor de jitter de 5 milisegundos, que junto con las gráficas de retardo obtenidas, demuestran un comportamiento muy similar entre las dos topologías. No obstante, en algunos casos, IP presenta niveles que superan los 14 ms, situación que no ocurre en MPLS.
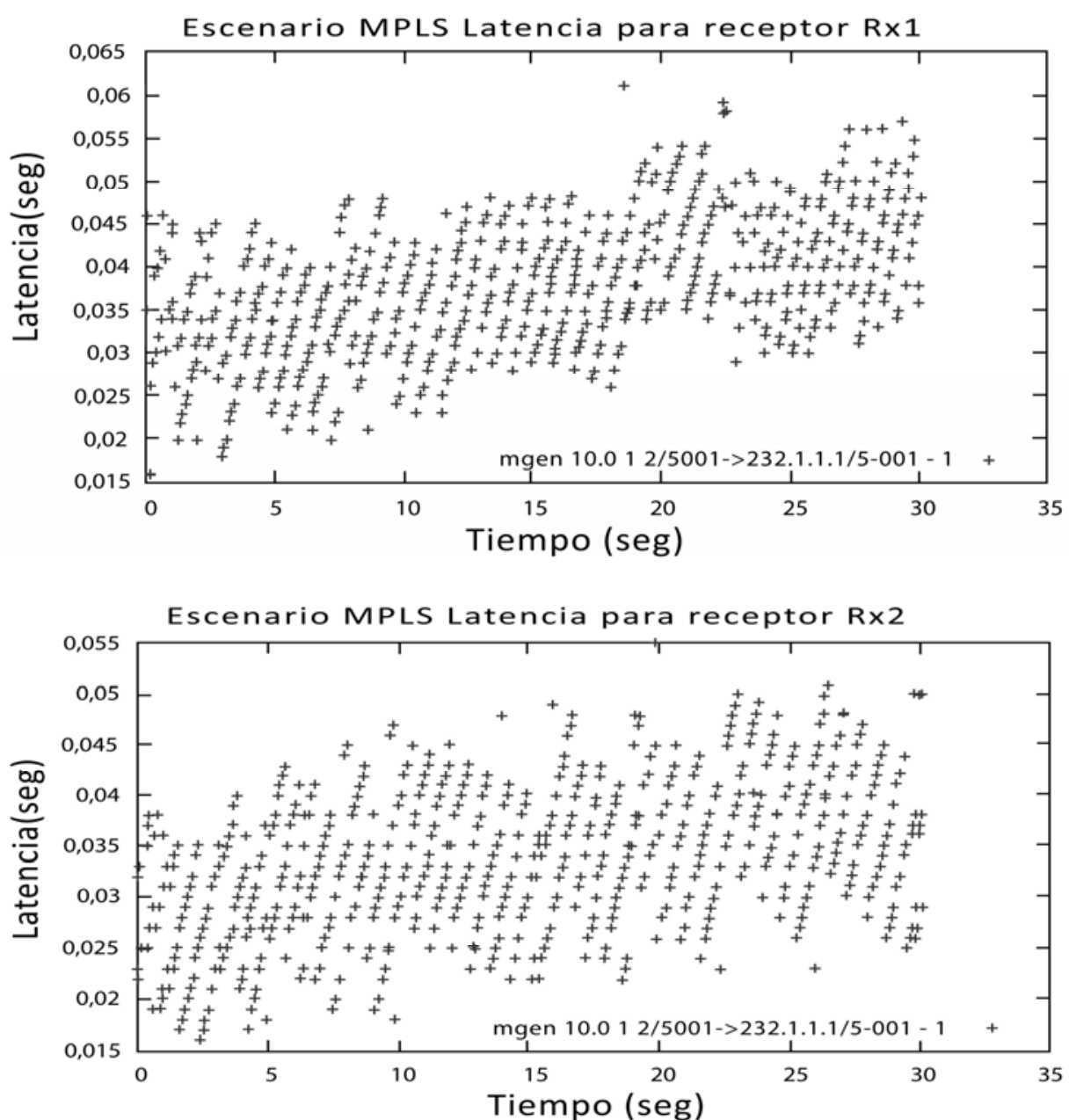

Fig. 5: Latencia entre la fuente y los receptores multicast $\mathrm{Rx1}$ y $\mathrm{R} \times 2$ para la red MPLS.

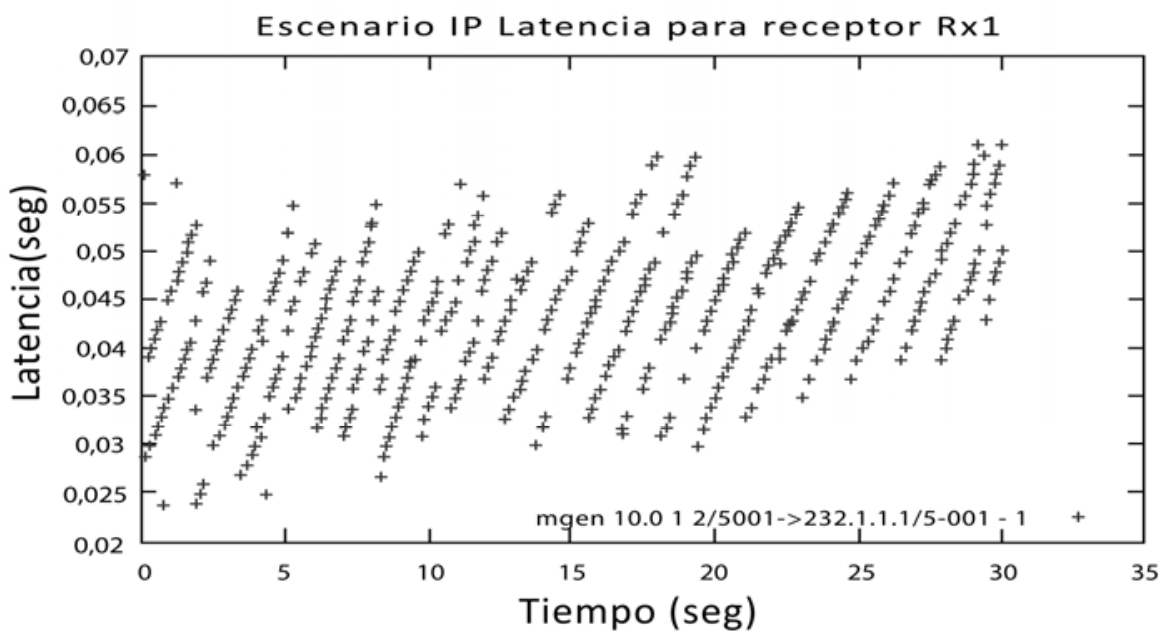




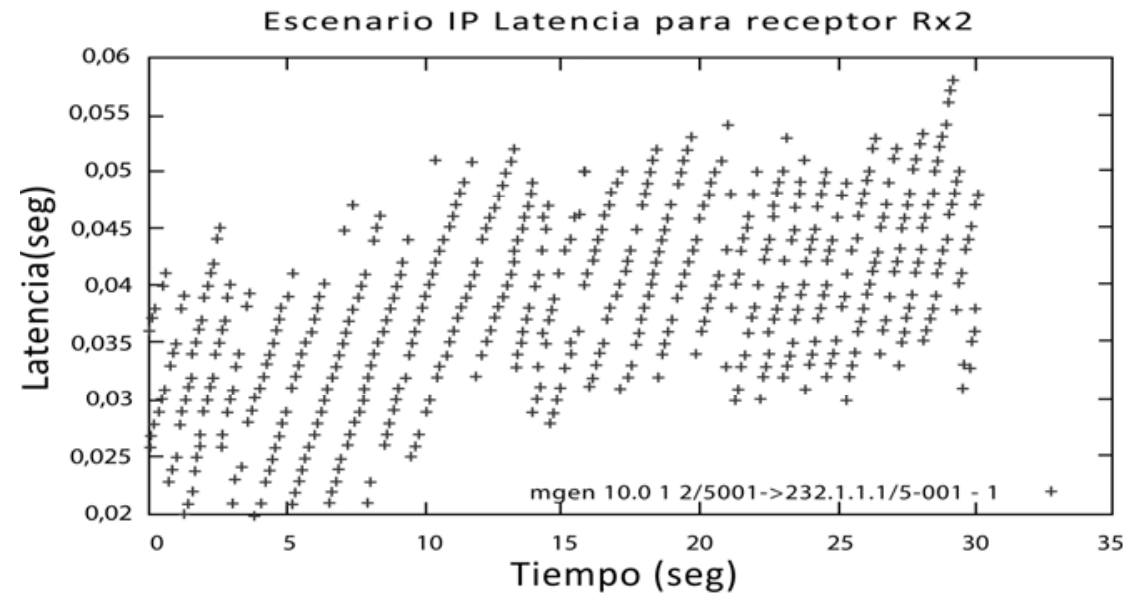

Fig. 6: Latencia entre la fuente y los receptores multicast $\mathrm{R} \times 1$ y $\mathrm{R} \times 2$ para la red PIM-DM.

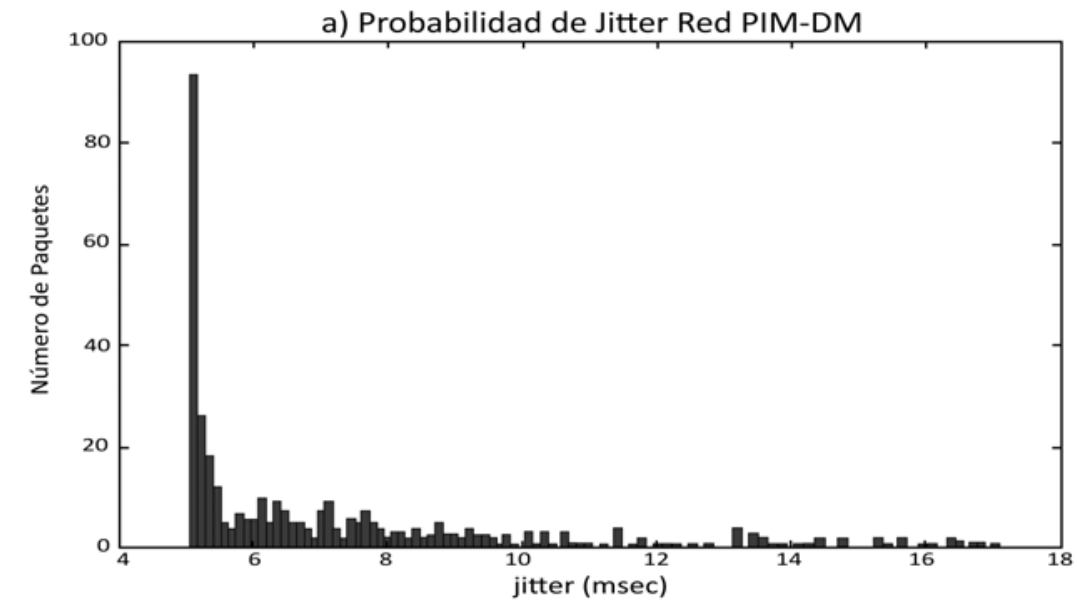

b) Probabilidad de Jitter Red MPLS

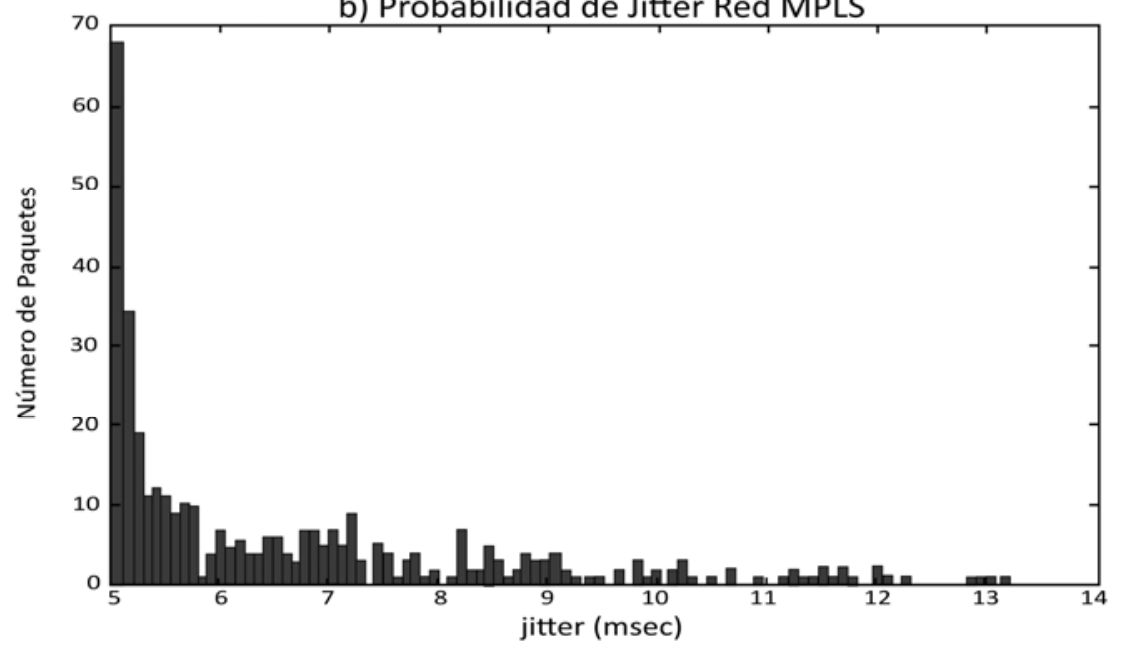

Fig. 7: Probabilidad de Fluctuaciones: a) Red PIM-DM y b) Red MPLS

\section{CONCLUSIONES}

El protocolo de señalización de reserva de recursos con ingeniería de tráfico permite incrementar la robustez de la tecnología MPLS ya que le puede brindar el soporte necesario para la transmisión de aplicaciones en entornos multicast garantizando una reserva de recursos de acuerdo con las necesidades exigidas (baja latencia y fluctuación, alta disponibilidad, ancho de banda) por aplicaciones como video y voz. 
La conmutación de etiquetas multiprotocolo con soporte para la utilización del estándar RSVP-TE es una alternativa muy interesante de re-encaminamiento de flujos multicast interactivos ante la caída de rutas principales ya que no solamente permite el establecimiento de caminos secundarios eficientes si no que incluye la posibilidad de aplicar criterios de ingeniería de tráfico, variable de red muy deseada por los proveedores de servicios de Internet.

Las mediciones de fluctuación y retardo, aunque mostraron comportamientos cercanos en ambos escenarios, siempre se caracterizaron por un mejor desempeño en el escenario MPLS. Esto se debe al distinto procesamiento que ejecutan los nodos en cada red: mientras que MPLS realiza una conmutación rápida de etiquetas a nivel de capa de enlace, el enrutamiento IP se realiza en la capa de red, lo que incluye un retardo adicional en el reenvío de paquetes. Con redes de mayor tamaño, estas diferencias se incrementarán, lo que demuestra que MPLS permite ofrecer mejor calidad de servicio.

\section{REFERENCIAS}

Amir, Y., Danilov, C., Goose, S., Hedqvist, D. Hedqvist., Terzis, A, An overlay architecture for highquality VoIP streams, IEEE Transactions on Multimedia 8 (6) 1250-1262 (2006).

Hachimi, M. y A. Abouaissa, MPLS based Approach for heterogeneous and scalable multicast in diffServ, Local Computer Networks, Proceedings 2006 31st IEEE Conference on, 515 - 518, Tampa, FL, USA, 14 al 16 de November (2006).

Hailong, M., G. Yunfei y Z. Jianwei, Fast reroute upon multi-homed domains Link Failures, ICIS '09 Proceedings of the 2009 Eigth IEEE/ACIS International Conference on Computer and Information Science, 183 - 187, Shanghai, China, 1 al 3 de Junio (2009).

Islam, S. y J. Atwood, The Internet group management protocol with access control (IGMP-AC), Local Computer Networks, Proceedings 2006 31st IEEE Conference on, 475 - 482, Tampa, FL, USA, 14 al 16 de November (2006).

Lodi, G., Panzieri, F., Rossi, D., Turrini, E, SLA-driven clustering of QoS-aware application servers, IEEE Transactions on Software Engineering 33 (3) 186-197 (2007).

Metz, Ch, Multiprotocol label switching and IP, part 2: Multicast virtual private networks, Internet Computing, IEEE, 10, 76 - 81, CA, USA, January to February (2006).

Networks and communication systems branch. Multi-Generator (en línea), (2008), disponible en: http://cs.itd.nrl.navy.mil/work/mgen/. Consultado en Octubre 15 de 2011.

Sung, Y. y Y. Lin, IPsec-based VolP performance in WLAN environments, IEEE Internet Computing ( 2008 ), 12, 77 - 82, Universidad Chiao Tung., Hsinchu, November to December (2008).

Thomas, M.S y I. Ali, Reliable, fast, and deterministic substation communication network architecture and its performance simulation, IEEE Transactions on Power Delivery 25 (4) 23642370. (2010)

Yuanzhe, X. y L. Chin-Tau, Network-Coding Multicast Networks with QoS Guarantees, Networking, IEEE / ACM Transactions on, 19(1), 265 - 274, Hong Kong, China, February (2011). 